\title{
Gauge potential singularities and the gluon condensate at finite temperatures
}

\author{
K. Langfeld ${ }^{\mathrm{a} *}$, E.-M. Ilgenfritz ${ }^{\mathrm{ab}} \dagger$, H. Reinhardt ${ }^{\mathrm{a}}$ and G. Shin ${ }^{\mathrm{ac}} \S$

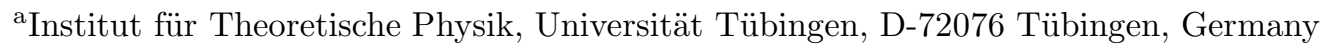 \\ ${ }^{\mathrm{b}}$ Research Center for Nuclear Physics, Osaka University, Osaka 567-0047, Japan \\ ${ }^{\mathrm{c}}$ Center for Theoretical Physics, Seoul National University, Seoul 151-742, Korea.
}

The continuum limit of $S U(2)$ lattice gauge theory is carefully investigated at zero and at finite temperatures. It is found that the continuum gauge field has singularities originating from center degrees of freedom being discovered in Landau gauge. Our numerical results show that the density of these singularities properly extrapolates to a non-vanishing continuum limit. The action density of the non-trivial $Z_{2}$ links is tentatively identified with the gluon condensate. We find for temperatures larger than the deconfinement temperature that the thermal fluctuations of the embedded $Z_{2}$ gauge theory result in an increase of the gluon condensate with increasing temperature.

\section{INTRODUCTION}

A precise definition of a Quantum Field Theory (QFT) is provided by the critical limit of a lattice model. Thereby, the QFT is solely specified by the number of space-time dimensions and symmetries. Here, we will critically re-investigate the lattice gauge theory with Wilson action which is assumed to reduce to continuum $S U(2)$ YangMills theory in the critical limit.

It is usually assumed that all $S U(2)$ link variables $U_{\mu}(x)$ can be expanded in the vicinity of the unit element for sufficiently small lattice spacing $a$, i.e. $U_{\mu}(x)=\exp \left\{i W_{\mu}(x) a\right\}$, such that the Wilson action density reduces to the Yang-Mills Lagrangian

$$
\frac{\beta}{2} \operatorname{tr}\left\{1-P_{\mu \nu}[U]\right\} \rightarrow \frac{a^{4}}{2 g^{2}} F_{\mu \nu}^{b}[W](x) F_{\mu \nu}^{b}[W],
$$

where $\beta=4 / g^{2}, P_{\mu \nu}[U](x)$ is the plaquette calculated in terms of the link elements $U_{\mu}(x)$ and

\footnotetext{
*talk presented by KL.

${ }^{\dagger}$ E.-M.I. thanks for the support by the Ministry of Education, Culture and Science of Japan (Monbu-Kagaku-sho) and for a grant to visit CERN.

$\ddagger_{\text {supported by DFG RE856/4-1 }}$

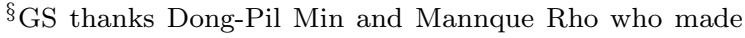
this collaboration possible.
}

$F_{\mu \nu}^{b}[W]$ is the usual field strength tensor. We will find that this Taylor expansion is not always justified. We will propose to relate the action density of the corresponding singularities to the gluon condensate.

\section{THE LATTICE THEORY OF GAUGE POTENTIAL SINGULARITIES}

Before we can localize the links where the above expansion eventually fails, we must bring the link elements as close as possible to the unit element by exploiting the gauge freedom $\Omega=\prod_{x} \omega_{x}$. We are thus led to implement an algorithm which puts the Monte Carlo configurations into the Landau gauge, accomplishing $\sum_{x, \mu} \operatorname{tr} U_{\mu}^{\Omega}(x) \rightarrow$ max, which is most suitable for our purpose. We have used an improved simulated annealing algorithm in order to find the maximum of the gauge fixing functional (details will be presented elsewhere). We then decompose the appropriately gauged link elements $U^{\Omega}$ into a center part and a coset part

$U_{\mu}^{\Omega}(x)=Z_{\mu}(x) \exp \left\{i A_{\mu}^{b}(x) t^{b} a\right\}$ 


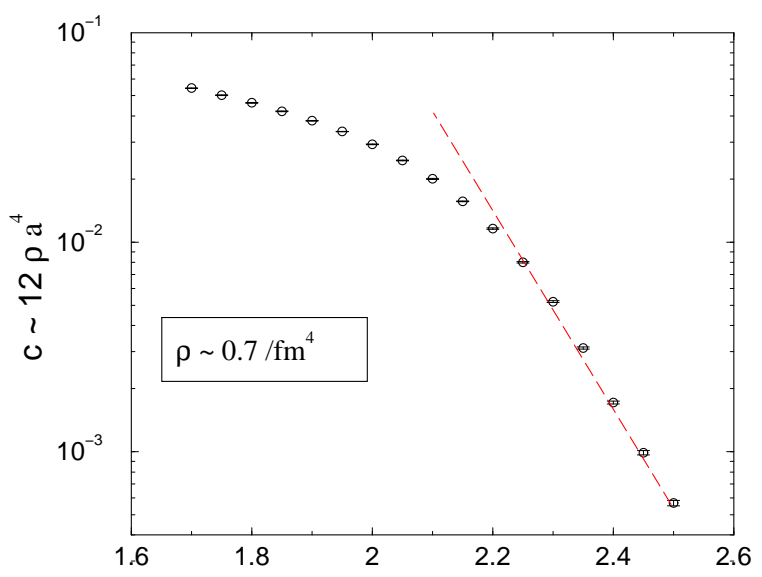

Figure 1. Density of non-trivial $Z_{2}$ plaquettes.

with $Z_{\mu}(x)=\operatorname{sign} \operatorname{tr} U_{\mu}^{\Omega}(x)$, and interpret $A_{\mu}^{b}(x)$ as the Yang-Mills gauge field in the continuum limit [1]. An expansion in powers of $a W_{\mu}$ (11) is uniformly justified only if the embedded $Z_{2}$ gauge theory spanned by the link elements $Z_{\mu}(x)$ is trivial, i.e. if $Z_{\mu}(x)=1, \forall x, \mu$. For a numerical check how many links $U_{\mu}(x)$ violate this condition it is possible directly to use the center action as singularity counter

$c:=\left\langle 1-\frac{1}{2} \operatorname{tr} P_{\mu \nu}[Z](x)\right\rangle$,

with the plaquettes $P_{\mu \nu}[Z](x)$ expressed in terms of the center projected configuration. For the Landau gauge, one expects that the non-trivial center elements $Z_{\mu}(x)=-1$ are sufficiently dilute close to the continuum limit. An isolated non-trivial center element generates 6 negative plaquettes each contributing 2 units to the center action in (3). In this dilute gas approximation, we therefore find $c \approx 12 \rho a^{4}$, where $\rho$ is the density of links carrying non-trivial center elements. Our numerical result for $c$ as function of $\beta$ is shown in figure 11. As expected, the singularity counter $c$ rapidly decreases with increasing $\beta$. Note, however, that the density $\rho$ of non-trivial center elements in physical units scales towards the continuum limit and is non-vanishing in the limiting case.

\section{THE FINITE TEMPERATURE GLUON CONDENSATE}

The Operator Product Expansion (OPE) somewhat artificially distinguishes between contributions from perturbative gluons and "other" contributions to Greenfunctions, which are not further specified. In lattice regularization, the perturbative gluon contribution is recovered by expanding the link variables (in Landau gauge) around the unit elements. Thus, the singular component, originating from the non-trivial center elements, contributes to the "other parts" which are parameterized by the condensates of the OPE. In particular, the gluon condensate is defined as the expectation value of the action density where the (divergent) perturbative gluon contribution has been subtracted. In practice, this subtraction uses the result of a high order calculation in lattice perturbation theory [2]. Alternatively, the gluon contribution can be removed by a cooling procedure which reduces the action of the coset (gluon) fields $A_{\mu}(x)$ [1]. The latter approach suggests that the gluon condensate $G$ gets contributions from the energy density stored in the underlying $Z_{2}$ fields, which are revealed by the coset cooling mechanism. We therefore find

$G a^{4} \propto c=12 \rho a^{4}$.

It was already observed above (see figure 1) that $G$ is lattice spacing independent and nonvanishing close to the continuum limit.

Let us now study the temperature dependence of the gluon condensate $G$ which is defined by (4) and (3). Temperature is introduced by varying the number $N_{t}$ of grid points in time direction, $T=1 / N_{t} a(\beta)$. We used the one loop formula for $a(\beta)$ and the string tension $\sigma=(440 \mathrm{MeV})^{2}$ as the reference scale. Simulations were performed on $16^{3} \times N_{t}, N_{t}=4 \ldots 16$ lattices for $\beta=2.3,2.4,2.5$. Due to the space-time asymmetry induced by the finite temperature, it is convenient to independently measure the spatial and the time-like parts of the gluon condensate, i.e. $G_{s}$ and $G_{t}$. These condensates are calculated from (41) where $c$ (3) is separately evaluated with spatial-spatial and spatial-timelike plaquettes, respectively. The result is shown in figure.2. 


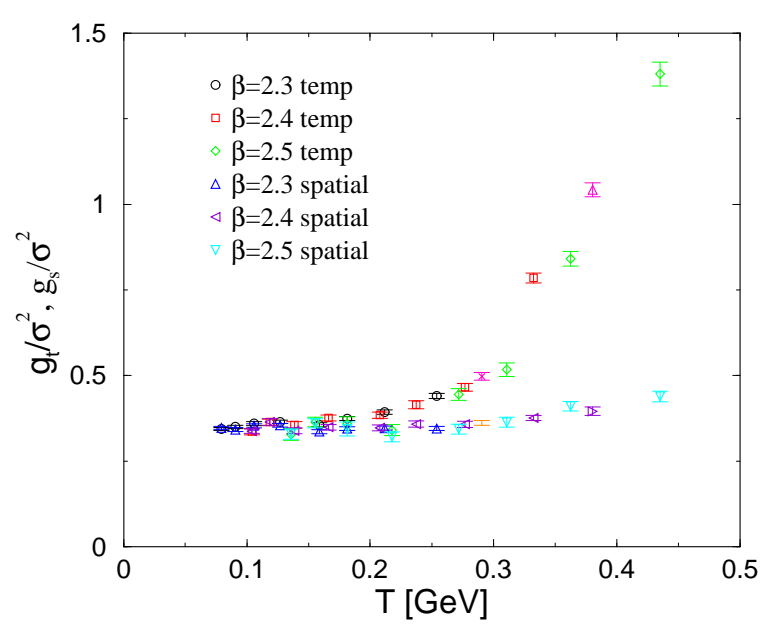

Figure 2. The condensate as function of temperature.

Below the deconfinement temperature for $S U(2)$ gluondynamics, $T_{c} \approx 300 \mathrm{MeV}$, the condensates $G_{s}$ and $G_{t}$ are weakly temperature dependent. Above $T_{c}$, the time-like component $G_{t}$ is rapidly increasing with $T$ while the variations of $G_{s}$ with $T$ are still moderate.

We interpret this behavior as follows: at zero temperature, the vacuum energy density of the underlying $Z_{2}$ gauge fields generates the gluon condensate. In the deconfined phase at high temperatures, these fields start thermally fluctuating in addition to the fluctuations of the coset (gluon) fields. The thermal energy density stored in the $Z_{2}$ system induces the rise of the gluon condensate with temperature while the gluonic black body radiation does not contribute by definition.

\section{DO WE HAVE TO REVISE THE CONTINUUM FORMULATION?}

The ab initio continuum formulation is based on the functional integral over gluon fields. A choice of the boundary conditions for gluon fields $A_{\mu}^{b}(x)$ is included in the definition of the QFT. If we wish to consider singularities of the gauge potential, the positions of these singularities are cut out from the space-time manifold. This procedure generates new boundaries at which we must specify appropriate conditions for the gauge potential. Different choices of the singularities might generate theories with different physical contents.

Let us illustrate this point in the familiar case of QED. A base manifold which is punctured at a closed line of space time can support a smooth gauge field which describes a magnetic monopole world line (Wu-Yang construction). Choosing several "singular lines" which are cut out from the base manifold, providing boundary conditions at all cuts and solving the QED partition function would result in a theory of photons moving in a background of given monopole world lines, but does not serve as a theory of monopoles. By contrast, the continuum limit of lattice compact QED represents a complete theory of photons and monopoles [3].

\section{CONCLUSIONS}

Landau gauge fixing of $S U(2)$ lattice gauge theory reveals a $Z_{2}$ vacuum texture. In the continuum limit, this texture consists of point-like gauge potential singularities with a density of $\rho \approx 0.7 / \mathrm{fm}^{4}$. The action density which is stored in the embedded $Z_{2}$ gauge system is identified with (part of) the gluon condensate. At temperatures above the deconfinement transition we observed that the $Z_{2}$ system carries thermal energy density leading to a gluon condensate which increases with temperature.

\section{REFERENCES}

1. K. Langfeld, E.-M. Ilgenfritz and H. Reinhardt, Nucl. Phys. B 608, 125 (2001) heplat/0008017.

2. G. Burgio, F. Di Renzo, G. Marchesini and E. Onofri, Phys. Lett. B422, 219 (1998).

3. J. Jersak, C. B. Lang and T. Neuhaus, Phys. Rev. Lett. 77, 1933 (1996) hep-lat/9606010. 